\title{
ESTILO COGNOSCITIVO Y SU IMPORTANCIA PARA LA ENSEÑANZA DE LA CIENCIA
}

\author{
NIAZ, $M$. \\ Departamento de Química, Escuela de Ciencias. Universidad de Oriente. Cumana, Estado Sucre. Venezuela.
}

(Trabajo presentado en el Encuentro Internacional de Educadores, Pedagogia'86, La Habana, Enero 1986)

\section{INTRODUCCION}

\section{SUMMARY}

This paper tries to look into the field depenleve of students' performance in Piagetians tasks based on different types of reasoning. The results show that over $50 \%$ of introductory courses of Chemistry, Physics, Mathematics and Biology are field dependent.

\section{INTRODUCCION}

Las tareas piagetianas de diferentes tipos de razonamiento podrian contener Efectos de Campo (Witkin, et. al. 1962). Se ha planteado (Karplus, Karplus y Wollman, 1974) que muchos estudiantes dejan de utilizar el razonamiento proporcional, ante una respuesta su* gerida por la manera particular como se presenta la tarea (Campo). De acuerdo a Witkin, esta preferencia personal refleja el Estilo Cognoscitivo del sujeto, y no necesariamente la etapa piagetiana de desarrollo cog. noscitivo. La percepción de un sujeto Dependiente de Campo está dominada por la organización global del Campo y relativamente hay poca posibilidad de percibir las partes discretas de un Campo, como tal (Witkin, Goodenough y Karp, 1967). Así mismo un sujeto Independiente de Campo trata de reestructurar la organización impuesta por el Campo, de acuerdo a sus criterios. Lawson (1976) señala que para el desarrollo del razonamiento formal se hace necesario una «estruc* turación analítica» de la información suministrada y una estructura (armazón) conceptual altamente articulada. Los sujetos Dependiente de Campo tienen deficiencias en estas habilidades.

Este estudio trata de investigar el Efecto de Campo sobre el rendimiento de los estudiantes en tareas piagetianas basadas en diferentes tipos de razonamiento, importante para la Enseñanza de la Ciencia (nivel secun. dario y primeros años de la universidad). Varios estu* dios (Lawson y Renner, 1974; Wollman, 1977; Kuhn y Brannock, 1977; Levine y Linn, 1977; Wollman y Lawson, 1978; Lawson 1982a) han demostrado la importancia de diferentes tipos de razonamiento (proporcional, combinatorio, probabilistico, control de variables, desplazamiento de volumen y conservación de peso) para cursos introductorios de química, física, matemática y biología. Lawson (1982b) obtuvo un coeficiente de correlación Pearson, $r=0,49(p=0,002)$ entre razonamiento formal de Piaget y Dependencia/ $\mathrm{In}$. dependencia de Campo. Así mismo en un estudio rea. lizado por Niaz y Lawson (1985) se encontró un coefi. ciente de correlación, $r=0,48(p<0,01)$ entre una prueba de balanceo de ecuaciones químicas y Depen. diencia/Independencia de Campo.

La primera parte de esta investigación (Niaz, 1985a) estudió el Efecto de Campo sobre el razonamiento proporcional y consistió en la aplicación de las siguientes pruebas a 318 estudiantes de Cursos Básicos (Ciencias y afines) de la Universidad de Oriente: a) Prueba de Figuras Encajada (PFE) de Witkin, et. al. 1971; b) Prueba de Razonamiento Proporcional (Lawson, 1978; Niaz, 1985b) de 9 items. Los resultados demuestran que los coeficientes de correlación de Pearson entre los 9 items de razonamiento proporcional y la PFE, fueron significativos $(r=0,19$ a 0,$39 ; p=0,001)$. Asi mismo, el coeficiente de correlación entre la calificación total de los 9 items de razonamiento proporcional y PFE, fue $r=0,50(p=0,001)$. 
El objetivo de esta segunda parte de la investigación fue estudiar el efecto de Campo sobre el rendimiento de los estudiantes en tareas Piagetianas basadas en los siguientes tipos de razonamiento: control de variables, desplazamiento de volumen, conservación de peso y relaciones espaciales.

\section{METODO}

\section{Sujetos}

El estudio se basó en 318 sujetos inscritos en 9 secciones de Química I (010-1114) en la Universidad de Oriente, Venezuela, durante el primer semestre de 1984. Edad $\overline{\mathrm{X}}=18,4$ años; $\mathrm{SD}=1,7$.

\section{Diseño Experimental y Procedimiento}

Durante la primera semana del semestre a todos los sujetos (Ss), les fueron aplicadas las siguientes pruebas: a) Prueba de Figuras Encajadas (PFE) de Witkin, et. al. (1971) para determinar el Estilo Cognoscitivo de los Ss, quiere decir, Dependencia (DC) / Independencia (IC) de Campo. El coeficiente de confiabilidad de la prueba con la presente muestra es de 0,79. b) Prueba basada en los siguientes 9 items (ver anexo): Cuatro items de Control de variables (CV1, CV2, CV3, y CV4); un item de Desplazamiento de Volumen (DV); tres items de Conservación de Peso (CP1, CP2 y CP3) un item de Relación Espacial (RE), Los items CV1, CV2, CV3, CV4, CPI y DV, con ciertas modificaciones fueron tomados de Lawson (1978). Asi mismo los items CP2, CP3 y RE fueron tomados de Niaz (1985b).

\section{RESULTADOS Y DISCUSION}

En la prueba de Figuras Encajadas (PFE), de Witkin, 179 Ss obtuvieron una calificación entre 0 a 6 , y utilizando los criterios de Witkin, fueron clasificados como Dependientes de Campo (DC). 109 Ss obtuvieron una calificación entre 7 a 12 , y fueron clasificados como intermedio (IM), y 30 Ss con una calificación entre 13 a 18, fueron clasificados como Independientes de Campo (IC). Un sujeto (IC), respondió correctamente a todos los cuatro items de control de variables. 91 $\mathrm{Ss}(\mathrm{IC}=11 ; \mathrm{IM}=45 ; \mathrm{DC}=35)$ respondieron correctamente a todos los tres items de conservación de peso.

Item CVI (ver Tabla 1): Muchos de los sujetos que respondieron correctamente (Respuesta a), justificaron de la siguiente manera:

«Si queremos hacer este experimento, tenemos que tomar cuerdas de diferentes largos, que podnan ser: $(1,2)$ ó $(1,3)$, pero habría que tomar en cuenta el peso (deben ser iguales) y por ello utilizo la $(1,2))$. Es fnctible que sujetos IC tengan una ventaja en comparación con los Ss DC, para descartar la respuesta $(1,3)$ como irrelevante. Se puede observar que de los 32 sujetos que respondieron $\mathrm{b}(1,3), 18(56 \%)$ eran $\mathrm{DC}$ y $4(13 \%)$ IC. $47 \%$ (14 de los 30) de los Ss IC y 13\% (23 de los 179) de los sujetos DC descifraron la información correctamente, quiere decir respondieron correctamente a $(1,2)$.

Item CV2 (ver Tabla 2): En este item el sujeto tiene que determinar si el tiempo necesario para que el péndulo

Tabla I

Relación entre Dependencia de Campo (DC), Independencia de Cam. po (IC) y Respuestas de los sujetos en el Item de Control de Variabjes CVI $(\mathrm{N}=318)$

\begin{tabular}{|c|c|c|c|c|}
\hline \multirow{2}{*}{ DESPLISTA } & \multirow{2}{*}{$N$} & \multicolumn{3}{|c|}{ NOHLRO DE SOJELOS } \\
\hline & & IC $(A=30)$ & IN $(N-109)$ & DC $(k=179)$ \\
\hline a & 74 & $14(19)^{*}$ & $37(50)$ & $23(31)$ \\
\hline $\mathrm{a}^{* *}$ & 62 & $7(11)$ & $17(27)$ & $38(61)$ \\
\hline$b$ & 32 & $4(93)$ & $10(31)$ & $26(56)$ \\
\hline c & 44 & - & $11(25)$ & $33(75)$ \\
\hline d & 24 & $2(\theta)$ & $6(25)$ & $16(67)$ \\
\hline e & 33 & $2(6)$ & $B(24)$ & $23(70)$ \\
\hline$s$ & 49 & $\pm(2)$ & $20(41)$ & $28(57)$ \\
\hline
\end{tabular}

- Las cifras en paréntesis representan porcentajes

" Sin justificación

f No contestó 
oscile entre los dos extremos, depende del peso que lleva la cuerda, manteniendo la longitud de la cuerda constante. $47 \%$ (14 de los 30 ) de los Ss IC y solamente $10 \%$ (17 de los 179) de los sujetos DC, respondieron correctamente: $C(2,3)$. Aparentemente los sujetos DC sienten la necesidad de variar el peso, para estudiar su efecto sobre la frecuencia de oscilación, pero no controlan la otra variable, que es el largo de la cuerda. Se puede observar que de los 40 sujetos que respondieron $b(1,3)$ $25(63 \%)$ eran DC y solamente $1(3 \%)$ IC.

Tabla II

Relación entre Dependencia de Campo (DC), Independencia de Campo (IC) y Respuestas de los Sujetos en el Item de Control de Variables CV2 $(\mathrm{N}=318)$

\begin{tabular}{|c|c|c|c|c|}
\hline \multirow{2}{*}{ RESPUESTA } & \multirow{2}{*}{$\mathbb{N}$} & \multicolumn{3}{|c|}{ MMERO DE SUJETOS } \\
\hline & & IC $(N=30)$ & $I M(N=109)$ & $D C(N+179)$ \\
\hline$B$ & 69 & $2(3)^{*}$ & $20(29)$ & $47(68)$ \\
\hline$b$ & 40 & $1(3)$ & $14(35)$ & $25(63)$ \\
\hline$c$ & 65 & $14(22)$ & $34(52)$ & $17(26)$ \\
\hline$c^{* *}$ & 40 & $5(13)$ & $15(38)$ & $20(50)$ \\
\hline$d$ & 22 & $4(18)$ & $5(23)$ & $13(59)$ \\
\hline e & 28 & $2(7)$ & $4(14)$ & $22(79)$ \\
\hline I & 54 & $2(4)$ & $17(31)$ & $35(65)$ \\
\hline
\end{tabular}

* Las cifras eri paréntesis representan porcentajes ** Sin justificación

f No contestó

Item CV3 (ver Tabla 3): Los Ss DC señalaron la necesidad de utilizar la metra $\mathrm{N}^{\circ} 2$, ya que se habia utilizado la metra $\mathrm{N}^{\circ} 1$, anteriormente. Se puede observar que $55 \%$ (98 de los 179) de los Ss DC, respondieron $\mathrm{b}$ (metra $\mathrm{N}^{\circ} 2$ ). Es interesante notar que $33 \mathrm{Ss}$ respondieron de la siguiente manera: «Se puede utilizar, cualquiera de las dos metras, lo importante es que sea lanzada desde la posición A». Esta respuesta no estaba incluida en la prueba.

Item $C V 4$ (ver Tabla 4): De los 10 Ss que respondieron correctamente (respuesta b) 7 eran DC y I, IC. Se puede observar que un número considerablemente grande (113) de Ss respondieron (b), sin dar una justificación adecuada. Es interesante set̉alar que 54 sujetos respondieron c (Necesita más información) y dieron la siguiente justificación: «Necesito saber si la metra metálica $\mathrm{N}^{\circ} 1$ pesa más que la metra metálica $\mathrm{N}^{\circ}$ 2 , o viceversa). En vista de que esa información era irrelevante para contestar el item, $56 \%$ (30 de los 54) de los sujetos DC respondieron $\mathrm{c}$.
Tabla IlI

Relación entre Dependencia de Campo (DC), independencia de Campo (IC) y Respuestas de los sujetos en el Item de Control de Variables CV1 $(\mathrm{N}=318)$

\begin{tabular}{|c|c|c|c|c|}
\hline \multirow{2}{*}{ FESPUESTA } & \multirow{2}{*}{ N } & \multicolumn{3}{|c|}{ WULERO DE SUJETOS } \\
\hline & & IC $(N=30)$ & IM $(\mathrm{N} \pm 10 \mathrm{~S})$ & $D C(N-179)$ \\
\hline$\approx$ & 25 & $4(16)^{*}$ & $10(60)$ & $11(44)$ \\
\hline $\mathrm{a}^{* * *}$ & 93 & $12(13)$ & $24(26)$ & $57(61)$ \\
\hline$b$ & 166 & $10(6)$ & $58(35)$ & $98(59)$ \\
\hline c & 34 & $4(12)$ & $17(50)$ & $13(38)$ \\
\hline
\end{tabular}

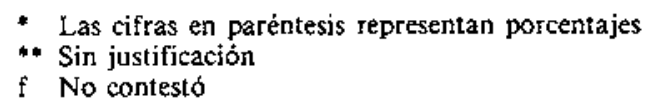

Tabla IV

Relación entre Dependencia de Campo (DC), Independencia de Campo (IC) y Respuestas de los sujetos en el Item de Control de Variables CV1 $(\mathrm{N}=318)$

\begin{tabular}{|c|c|c|c|c|}
\hline \multirow{2}{*}{ RESPJESTA } & \multirow{2}{*}{ \$ } & \multicolumn{3}{|c|}{ NOMERO DE SUJETOS } \\
\hline & & IC $(N=30)$ & $I M(N=109)$ & $D C(N=179)$ \\
\hline a & 77 & $5(6)^{*}$ & $30(39)$ & $42(55)$ \\
\hline b & 10 & $1(10)$ & $2(20)$ & $1(70)$ \\
\hline$+\infty$ & 113 & $12(11)$ & $38(34)$ & $63(56)$ \\
\hline c & 54 & $6(11)$ & $18(33)$ & $30(56)$ \\
\hline d & 64 & $6(9)$ & $21(33)$ & $37(58)$ \\
\hline
\end{tabular}

* Las cifras en paréntesis representan porcentajes

** Sin justificación

f No contestó

Item $D V$ (ver Tabla 5): $68 \%$ (217 de los 318 ) de los Ss respondieron (a), quiere decir el nivel de agua sube más en el caso del cilindro $B$, que del cilindro $A$. El item DV es un indicador del comienzo del razonamiento for. mal (Piaget, Inhelder y Szeminska, 1960). De acuerdo a Lawson, Blake y Nordland (1974), el sujeto debe cumplir con los siguientes requisitos para contestar correctamente: a) Debe comprender el concepto abstracto de desplazamiento del volumen; b) Debe eliminar una contradicción, ignorando la respuesta sugerida por la per. cepción que se encuentra en los pesos desiguales de los cilindros A y B. Al contrario se debe concentrar en igualdad del volumen de los dos cilindros. Se puede observar que la respuesta (a) sugerida por el Campo fue 
Tabla V

Rejación entre Dependencia de Campo (DC), Independencia de Campo (IC) y Respuestas de los sujetos en el Item de Control de desplazamiento de voltmen DV $(\mathrm{N}=318)$

\begin{tabular}{|c|c|c|c|c|}
\hline \multirow{2}{*}{ RESPJVSTA } & \multirow{2}{*}{$\mathbf{N}$} & \multicolumn{3}{|c|}{ NOMLEO DE SUJETOS } \\
\hline & & IC $(\sin 30)$ & $\ln (N=109)$ & $D C(N=179)$ \\
\hline a & 217 & $55(7)^{*}$ & $74(34)$ & $128(59)$ \\
\hline$b$ & 9 & - & $3(33)$ & $6(67)$ \\
\hline c & 55 & $10(56)$ & $22(40)$ & $23(42)$ \\
\hline$c^{* *}$ & 32 & $4(13)$ & $10(31)$ & $18(56)$ \\
\hline d & 5 & $1(20)$ & - & $4(80)$ \\
\hline
\end{tabular}

- Las cifras en paréntesis representan porcentajes

** Sin justificación

d No contestó

Campo fue aceptada por $72 \%$ (128 de los 179) de los sujetos DC, y $50 \%$ (15 de los 30 ) de los sujetos IC. Aunque los Ss IC, aceptaron la respuesta (a) a menos grado que los sujetos DC, el nivel de aceptación es bastante elevado. Así mismo solamente 10 de los 30 sujetos IC, contestaron correctamente, respuesta (c). Aparentemente no encontramos una explicación satisfactoria de estos resultados. El coeficiente de correlación (ver Tabla 10) $r=0,16 ; p=0,004$ entre PFE y el item DV es un indicador de que varios factores podnian incidir sobre el rendimiento. Es interesante señalar que los sujetos que respondieron (a) algunos dieron las siguientes justificaciones:

- «Porque a mayor peso la densidad de agua será mayor, es decir sube más».

- «A mayor masa habrá mayor empuje».

- «B tiene mayor volumen».

- «Agua desplazaba será en relación directa a la masa que posee el sólido».

- «B pesa más y por eso hay mayor presión sobre el agua, lo que hace que el nivel sea mayorn.

- «B tiene mayor densidad que A».

- «B tiene mayor masa y la gravedad lo atrae con mayor fuerza».

Item CPI (ver Tabla 6): La gran mayoría de los sujetos IC y DC respondieron correctamente: respuesta (b). Estos resultados se podrian interpretar de la siguiente manera: A medida que los sujetos hayan logrado el tipo de razonamiento en que se basa un item, el papel del Estilo Cognoscitivo es menos determinante (ver Tabla 10: coeficiente de correlación entre el item CPl y PFE, $\mathrm{r}=0,01 ; \mathrm{p}=0,432$ ).
Tabla VI

Relación entre Dependencia de Campo (DC), Independencia de Campo (IC) y Respuestas de los sujetos en el Item de Conservacion de Peso $\mathrm{CPl}(\mathrm{N}=318)$

\begin{tabular}{|c|c|c|c|c|}
\hline \multirow{2}{*}{ RESTVISS TA } & \multirow{2}{*}{$\mathbf{H}$} & \multicolumn{3}{|c|}{ NOMORO DE SUJEIOS } \\
\hline & & IC $(\mathrm{N}=30)$ & IN $(N-109)$ & DC $(15179)$ \\
\hline * & to & - & $4(40)^{*}$ & $6(60)$ \\
\hline$b$ & 302 & $29(10)$ & $103(34)$ & $170(56)$ \\
\hline e & 6 & $1(17)$ & 2(33) & $3(50)$ \\
\hline
\end{tabular}

- Las cifras en paréntesis representan porcentajes

Item $C P 2$ (ver Tabla 7): Se cree que una de las posibles respuestas sugeridas por el Campo es la (c). $82 \%$ (69 de los 84) de los sujetos que contestaron (c), eran DC, y $1 \%$ (1 de los 84 ) IC. Se observó que la gran mayoría de los Ss, sumaron todos los pesos suministra. $\operatorname{dos}(163,5+13,6+103,2=280,3)$, manifestando así una incapacidad de descifrar información relevante. Así mismo se puede observar que $77 \%$ (23 de los 30 ) de los sujetos IC, contestaron correctamente la respuesta (b).

\section{Tabla Vll}

Relación entre Dependencia de Campo (DC), Independencia de Campo (IC) y Respuestas de los sujetos en el "tem de Conservación de Peso CP2 $(\mathrm{N}=318)$

\begin{tabular}{|c|c|c|c|c|}
\hline \multirow{2}{*}{ Destoses } & \multirow{2}{*}{$\mathrm{N}$} & \multicolumn{3}{|c|}{ SOMLRO DE SUJETOS } \\
\hline & & IC $(N=30)$ & $I M(N-109)$ & $D C(x-179)$ \\
\hline a. & $\$ 1$ & $1(9)^{*}$ & $2(18)$ & e $(73)$ \\
\hline$b$ & 179 & $23(13)$ & $75(42)$ & $81(45)$ \\
\hline c & 84 & $1(1)$ & $14(17)$ & $69(82)$ \\
\hline d & 29 & $3(10)$ & $12(41)$ & $14(46)$ \\
\hline$\theta$ & 15 & $2(13)$ & $6(40)$ & $7(47)$ \\
\hline
\end{tabular}

- Las cifras en paréntesis representan porcentajes e No contestó

Item $C P 3$ (ver Tabla 8): Aparentemente las dos respuestas sugeridas por Campo son: (b) y (c). $75 \%$ (30 de los 40) de los Ss que respondieron (b), eran DC y ninguno IC. Así mismo $70 \%$ (32 de los 46) de los Ss que respondieron (c), eran DC y $7 \%$ (3 de Ios 46 ) eran IC. 
Tabla VIII

Relación entre Dependencia de Campo (DC), Independencia de CamDo (IC) y Respuestas de los sujetos en el Item de Conservación de Peso CP3 $(\mathrm{N}=318)$

\begin{tabular}{|c|c|c|c|c|}
\hline \multirow{2}{*}{$\begin{array}{l}\text { :RESFUESTA } \\
\vdots\end{array}$} & \multirow{2}{*}{$\mathrm{N}$} & \multicolumn{3}{|c|}{ NUNERO DE SDJETOS } \\
\hline & & IC $(x=50)$ & IM $(N=109)$ & $B C(N=179)$ \\
\hline a & 149 & $20(13)^{\prime \prime}$ & $64(43)$ & $65(44)$ \\
\hline$a^{* *}$ & 14 & - & $3(21)$ & $11(79)$ \\
\hline$b$ & 40 & - & $10(25)$ & $30(75)$ \\
\hline c & 46 & $3(7)$ & $11(24)$ & $32(70)$ \\
\hline$d$ & 29 & $3(10)$ & $9(3 y)$ & $17(59)$ \\
\hline e & 40 & $4(10)$ & $12(30)$ & $24(60)$ \\
\hline
\end{tabular}

- Las cifras en paréntesis representan porcentajes

* Sin justificación

e No cortestó

Ilem $R E$ (ver Tabla 9): $70 \%$ (21 de los 30) de los Ss IC, respondieron correctamente y $26 \%$ (46 de los 179) de los sujetos $\mathrm{DC}$, respondieron correctamente. Se puede observar que los Ss IC tienen mayor capacidad de visualizar y analizar, las relaciones en el espacio.

A continuación se presenta una distribución de los diferentes items utilizados en este estudio, de acuerdo a la etapa piagetiana y el porcentaje de los sujetos que respondieron correctamente (Tabla 10):
Tabla IX

Relación entre Dependencia de Campo (DC), Independencia de Campo (IC) y Respuestas de los sujetos en el Item de Relación Espacial $R E(N=318)$

\begin{tabular}{|c|c|c|c|c|}
\hline \multirow{2}{*}{ RDSFURSTA } & \multirow{2}{*}{$N$} & \multicolumn{3}{|c|}{ NOTJRO DE SDJETOS } \\
\cline { 3 - 5 } & & IC (N=30) & IM (N=109) & NC (N=179) \\
\hline \multirow{2}{*}{$*$} & 111 & $21(19)^{*}$ & $44(40)$ & $46(41)$ \\
$\mathrm{C}$ & 190 & $7(4)$ & $60(32)$ & $123(65)$ \\
& 17 & $2(12)$ & $5(29)$ & $10(59)$ \\
\hline
\end{tabular}

- Las cifras en paréntesis representan porcentajes

a Todas las cinco letras tespondidas correctamente b Menos de cinco letras respondidas correctamente c No contestó
Lawson (1978) realizó el Análisis de Componentes Prin cipales y encontró que los items de Control de Variables, tenían dos factores. Como era de esperarse, uno de estos factores $(0,65)$ era común con los demás items de rezonamiento formal: Proporcional $(0,75)$, Combi* natorio $(0,64)$ y Probabilístico $(0,77)$. El otro factor $(0,39)$ era el mismo que el del item Desplazamiento de Volumen $(0,82)$. Los resultados obtenidos en este estudio muestran que los items de Control de Variables (CV1 y CV2) tienen algunos rasgos similares (manejo

Tabla X

\begin{tabular}{|c|c|c|c|c|c|c|}
\hline \multirow[b]{2}{*}{ Item } & \multirow[b]{2}{*}{ Etapa Piagetiana } & \multicolumn{2}{|c|}{$\begin{array}{l}\text { que respondieron } \\
\text { amente }\end{array}$} & \multicolumn{3}{|c|}{$\begin{array}{l}\text { NQ de sujetos con } \\
\text { Estilo Cognoscitivo }\end{array}$} \\
\hline & & $\mathrm{N}$ & $(8)$ & IC & $\overline{I M}$ & $D C$ \\
\hline CPI & Operacional Concreta & 302 & (95) & 29 & 103 & 170 \\
\hline $\mathrm{CP} 2$ & Operacional Concreta & 179 & $(56)$ & 23 & 75 & 81 \\
\hline $\mathrm{CP} 3$ & operacional Concreta & 149 & (47) & 20 & 64 & 65 \\
\hline$R E$ & Operacional Concreta & 111 & (35) & 21 & 44 & 46 \\
\hline DV & Operacional Formal Temprana & 55 & $(17)$ & 10 & 22 & 23 \\
\hline CVI & Operacional Formal & 74 & (23) & 14 & 37 & 23 \\
\hline $\mathrm{C} 2$ & Operacional Formal & 65 & $(20)$ & 14 & 34 & 17 \\
\hline $\mathrm{C} 3$ & Operacional Formal & 25 & $(8)$ & 4 & 10 & 11 \\
\hline CV4 & Operacional Fommal. & 10 & $\langle 3\rangle$ & 1 & 2 & 7 \\
\hline
\end{tabular}


de largo y peso de la cuerda) con el item de Desplazamiento de Volumen. Este estudio en concordancia con los resultados reportados por Lawson (1978) mostró el rendimiento de los Ss muy similar, en los items: DV $(17 \%)$; CVI $(23 \%)$; y CV2 $(20 \%)$.

\section{CONCLUSIONES}

En este estudio se ha encontrado evidencia para apoyar la hipótesis de que los sujetos Dependientes de Campo son más afectados por los Efectos de Campo. Se obtuvieron coeficientes de correlación (ver Tabla 11) significativos entre la Prueba de Figuras Encajadas y los items de Control de Variables $(\mathrm{r}=0,28 ; \mathrm{p}=0,001)$; Desplazamiento de Volumen $(\mathrm{r}=0,16 ; \mathrm{p}<0,01)$; Conservación de Peso $(r=0,28 ; p=0,001)$ y Relación Espacial $(r=0,26 ; p=0,001)$. Es importante señalar que los coeficientes de correlación son significativos pero relativamente bajos, siendo esto un indicador del hecho que además del Estilo Cognoscitivo, otros factores cognoscitivos (Pascual-Leone, 1970; Case, 1978; Niaz y Lawson, 1985) inciden sobre el rendimiento de los estudiantes. Finalmente, se puede observar (Tabla 11) que los coeficientes de correlación entre la calificación total de los 9 items de razonamiento proporcional (Niaz, 1985a) y los items de Control de Variables $(r=0,41) ; p<0,01)$, Desplazamiento de Volumen $(r=$ $0,23 ; \mathrm{p}<0,01)$, Conservación de Peso $(\mathrm{r}=0,31 ; \mathrm{p}<$ $0,01)$, Relación Espacial $(r=0,23 ; p=0,001)$ y la Prueba de Figuras Encajadas $(r=0,50 ; p=0,001)$ fueron significativos.

En los últimos años varios investigadores (Griffiths, 1976; Shayer y Adey, 1981; Lawson y Snitgen, 1982) han enfatizado la importancia de los procesos piagetianos de razonamiento formal, para la enserfanza de la Ciencia. En vista de los resultados obtenidos en este estudio, indicando que muchas de las tareas piagetianas contienen Efectos de Campo, es necesario que los docentes consideren el papel jugado por el Estilo Cognoscitivo. Así mismo los resultados muestran que más del $50 \%$ (179 de los 318) de los estudiantes inscritos en cursos introductorios de química, física, matemática y biologia son Dependientes de Campo. Este hecho hace necesario que los docentes utilicen estrategias adecuadas, para disminuir el efecto de los factores perceptuales de Campo.

\section{Agradecimiento}

Este trabajo fue realizado con los fondos del Consejo de Investigación de la Universidad de Oriente $\left(\mathrm{N}^{\circ}\right.$ del Proyecto: CI-5-023-00235/84-85).

Tabla XI

Coeficientes de Correjación Pearson entre la Prueba de Fìguras Encajadas (PFE) y los Items de Control de Variables (CV), Desplazamiento de Volumen (DV), Conservación de Peso (CP) y Relación Espacial (RE). $N=318$.

\begin{tabular}{|c|c|c|c|c|c|c|c|c|c|c|c|c|c|}
\hline Vertable" & pre & $\mathrm{cyt}$ & $\mathrm{c} 2$ & $\mathrm{cv3}$ & $\mathrm{cv}_{4}$ & Total CV & $\mathrm{pv}$ & $C P 1$ & $\mathrm{CP2}$ & CP3 & Total CP & $\mathrm{RE}$ & Total ir \\
\hline $\mathrm{PFE}$ & $t$ & & & & & & & & & & & & \\
\hline $\mathrm{Cr}:$ & 0.27 & 1 & & & & & & & & & & & \\
\hline $\mathrm{cr} 2$ & 0.37 & 0,87 & 1 & & & & & & & & & & \\
\hline $\mathrm{cr} 3$ & $0,06^{8}$ & $0,18 *$ & $0,24^{*}$ & 1 & & & & & & & & & \\
\hline $\mathrm{Cv}_{4}$ & 0,00 & $0,20^{\circ *}$ & $0,13^{*}$ & $0,02^{b}$ & $t$ & & & & & & & & \\
\hline Total CV & $0,2 \vec{\theta}^{*}$ & $0.91 *$ & $0,9 *$ & $0,47^{*}$ & 0,34 & $\xi$ & & & & & & & \\
\hline bv & $0,16^{*}$ & $0,13^{\mathrm{a}}$ & $0.11^{\mathrm{a}}$ & $0,04^{\circ}$ & $0,05^{\circ}$ & $0,12^{\mathrm{a}}$ & 1 & & & & & & \\
\hline $\mathrm{CP} 1$ & $0,01^{b}$ & $0, \infty$ & $0, \infty$ & $0,01^{\circ}$ & $0,04^{3}$ & 0,00 & $0,05^{6}$ & 1 & & & & & \\
\hline $\mathrm{CP2}$ & $0,2 \pi$ & $0,15^{*}$ & $0,19^{*}$ & $0,08^{\mathrm{a}}$ & $0,05^{b}$ & $0,48^{*}$ & $0,12^{\mathrm{a}}$ & $0,06^{\circ}$ & $i$ & & & & \\
\hline $\mathrm{CP3}$ & 0,19 & $0,13^{*}$ & $0,12^{\mathrm{a}}$ & $0.14^{*}$ & $0,01^{b}$ & $0,16^{*}$ & $0,00^{8}$ & $0,07^{8}$ & $0,14^{*}$ & 1 & & & \\
\hline Tatel CP & $0,2 B^{-*}$ & $0,16^{*}$ & $0,17^{*}$ & $0,11^{a}$ & $0,05^{b}$ & $0,19^{*}$ & $0,14^{*}$ & $0,35^{*}$ & $0,75^{*}$ & $0.71^{*}$ & 1 & & \\
\hline $8 \varepsilon$ & $0.25^{*}$ & $0,12^{\mathrm{a}}$ & $0,09^{\mathrm{a}}$ & $0, \infty$ & $0,10^{\mathrm{a}}$ & $0,10^{\mathrm{a}}$ & $0.09^{\mathrm{a}}$ & 0,00 & $0,07^{\mathrm{E}}$ & $0,35^{*}$ & $0.13^{*}$ & 3 & \\
\hline Total 12 & $0,50^{* *}$ & 0,35 & $0,39^{\circ}$ & $0,17^{*}$ & $0,22^{*}$ & $0,41^{*}$ & $0,23^{*}$ & $0,05^{\circ}$ & $0,25^{\prime \prime}$ & 0,26 & $0.3 i^{\circ}$ & $0,23 *$ & 1 \\
\hline
\end{tabular}

"Total Pr = Califiosción totel obtentda en loa 9 itame de razonamtento proporolonei, ver N1az, 1985a.

${ }_{p}<0,01, \cdots p=0,001,{ }_{p}<0,1,{ }_{p}<<0.5$ 


\section{ANEXO}

1t暗自 OV1, CV2
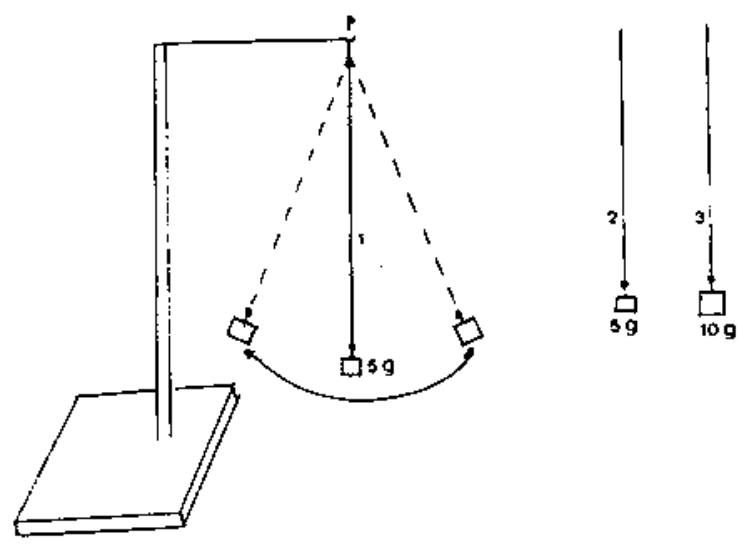

Trmasos tres cuerdas: 1,2 y 3 . 10 cuerda 1 es mis barga pue la 2 y

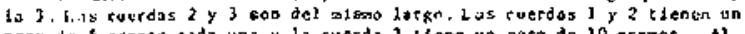
peso de 5 gramos cada una y la cuerda $j$ isene un pesa de 10 gramos. Al colsar una cuerdo del punto de apoyo, $P$, se puede formar un péndulo que puede ascilar entre los dos extremas, tal como lo denvestra en la figura.

Pregunta a, aieteros hacer un experimetico pata deccerminas si el t1erpo necesarto pars quc el pä́ndulo oseile entre los dos extreass, depende del

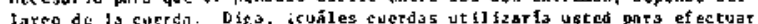
ril exper irenco?

Marque in cespausen corresta estin una " $x$ "
a) -1 y 2
d) - $1.2 \times 3$
b) $-3 \times 3$
e)- ?olamente

c) 2 y 3

Fregunta is. Querengs hacer un exper imento para determinar st cl cscopo necesario para que el pêndula oselle entre los dos cotrenos, depende del peso que 11 eva 1a cuerda. Diga, iCuáles querdas utilizarta vered para ef ectuar el experimenta?

Marput la respuesza sorrecto con uns " $x$ ".
a) - 1 y 2
d) $-1,2$ y 3
b). 1 y 3
e). 2 solamente
c). $2 \times 3$

Itew CVy

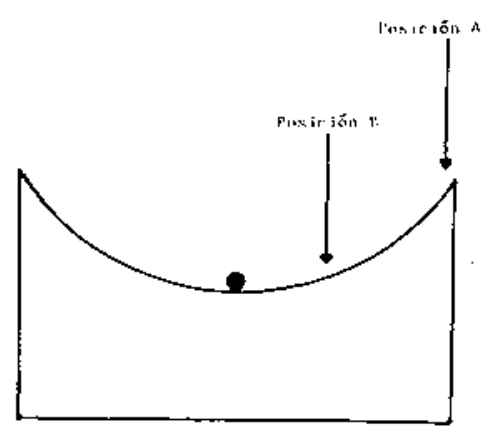

Mecrs $\$ 21 \oplus$

Aquí tenemos una ratog \{como lo usada por los patinadoress, con una metra

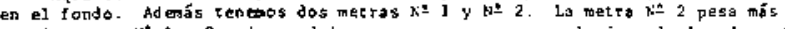
que 1a metrs ki 1. Cuando se deja caer suavemente a cualquiers de las dos me-

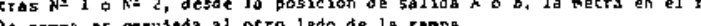
la rampa es copujodia al octo lado de la ramps.

Pregunta: En un experínento se dejó cacr sugvemente le metro kt 1 desde la posición B, y se observó que la metra en el fondo subio al otro lado de la ran

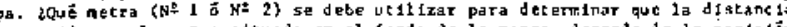
recorr1da por la metra strueda en el fondo da la ranpa, depende de la porlctön

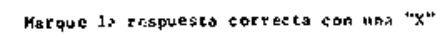

a) - Metra Nㅛ 1

b)- Yetra Nㅡㄹ 2
Itas $\mathrm{CP}_{4}$

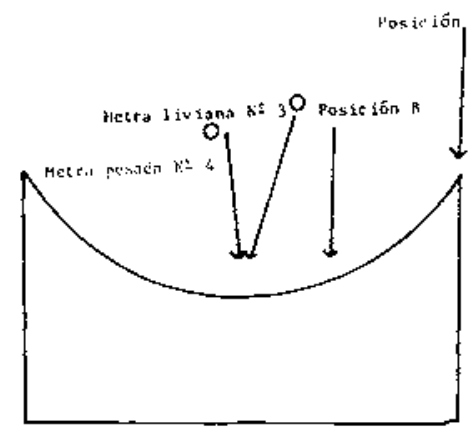

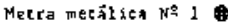
Metra metálica ke 20

Cono en el teen anterior, aquí teremos uns rasos. en el fondo de to twal se nodría colocar una metra liviana № 30 lo metrapesada ${ }^{\circ} 4$, adcoís tene-

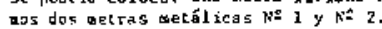

1 Experimento: Cuando se colocó la metra pesada $N^{*}$ a en el fondo de la rampa y se defó eacr suavenente lo metra setál lca $\mathrm{N}^{*} 1$, desde la posictón $A$, la aptra $\$ 2$

II Experimento: Cuando se colocó lo netra loviana 3 en el fondo de la rampa y 58 defó caer suavcrente 1 t toetro petálita $N^{*} 2$, desde la pọsición $A$, la perta $k=3$ en el fondo de la rampa subió al orro lado de la rapas Se

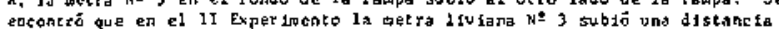
mayor que 1 a netxa pesada 326 , en el I Exper tento.

Presunta: ise puede concluir a portir de los experdocncos I y II, que la me-

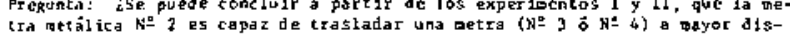
cancia que la wetra metalica $N=1$ ?

karque la respuesta corecesta con una "x".

a) 51

b).- $\therefore 0$

c)- Necesilta mä́s informac lớn

Itoe DY

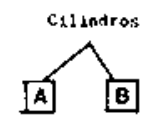

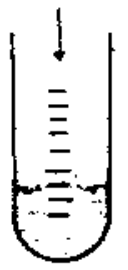

Vaso aln cllindro

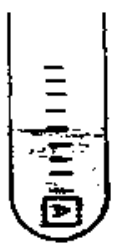

Yaso can c11 1ndro A
Tanes dos cilindros A I B, de la disma al cura g groost. A, pesa 25 gramos y B Tomeros dos cilindros A I B, de la disma alcura g grosot. A. pesa 25 gratos y B

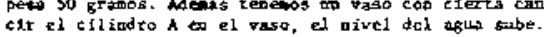

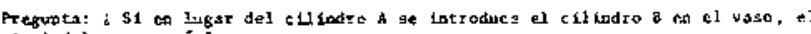

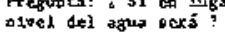

Yarque la respuesta correcta con una $*$ -

a) - ayor que to el caso ded celinito $A$

b) - hor que an el caso del cllindro a

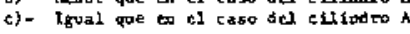

I te: CP:

En la 51 guiente f 1qui aprecen dos pelotas de plastilina del wigoo tamaino $y$ geso: $A$ B.
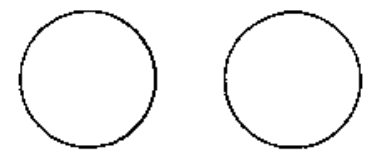

Inaginese que ahota a la pelota 8 se le da la fores de una arepes

Preguntil: i Quil de las dos, La pelote o la ereph, pesan más?

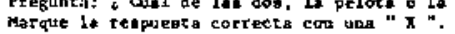

4) - Le pelote pesa más.

b)- Las dos peran iguales.

c) - La arepa peod tát. 


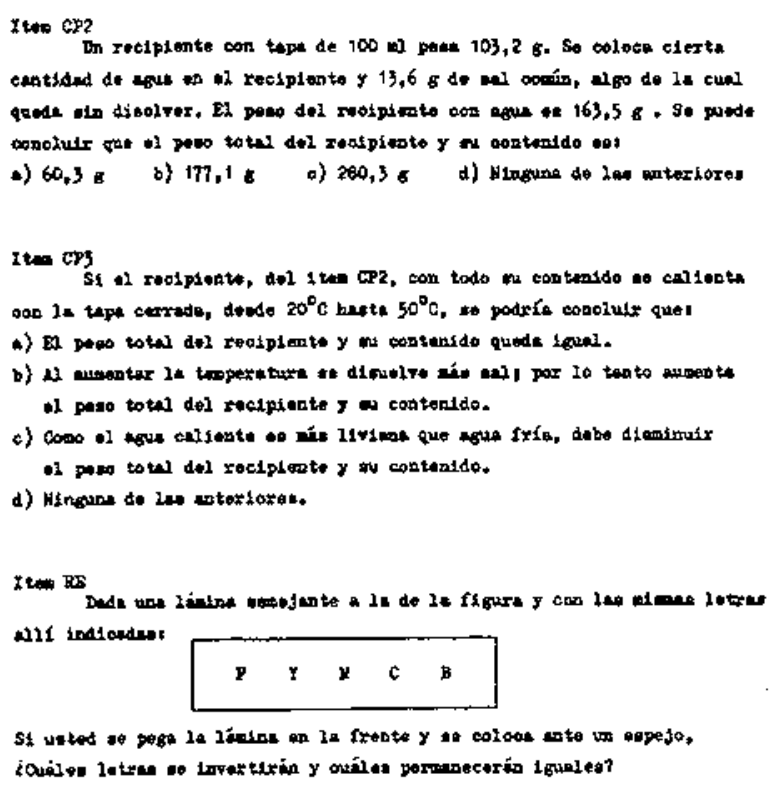

\section{REFERENCIAS BIBLIOGRAFICAS}

CASE, R., 1978, Piaget and beyond: Toward a developmentally based theory and technology of instruction, en Glaser, R. (Ed.) Advances in Instructional Psychology, (Lawrence Erlbaum: Hillsdale $(\mathrm{NJ})$ ).

GRIFFITHS, D.H., 1976, Physics Teaching: Does it hinder intellectual development? American Journal of Physics, 44(1), 81-85.

KARPLUS, E.F., KARPLUS, R. y WOLLMAN, W., 1974, Inteliectual development beyond elementary school IV: Ratio, the influence of cognitive style, School Science and Mathematics, 74(6), 476-482.

KUHN, D. y BRANNOCK, J., 1977, Development of the Isolation of Variables Scheme in Experimental and \& $\mathrm{Na}$ tural Experiment» Contexts, Developmental Psychology, 13(1), 9-14.

LAWSON, A.E., 1976, Formal operations and field independence in a heterogeneous sample, Perceptual and Motor Skills, 42, 981-982.
LAWSON, A.E., 1978, The development and validation of a classroom test of formal reasoning, Journal of Research in Science Teaching, 15(1), 11-24.

LAWSON, A.E., 1982a, The nature of advanced reasoning and science instruction, Journal of Research in Science Teaching, 19, 743-760.

LAWSON, A.E., 1982b, Formal reasoning, achievement and intelligence: an issue of importance, Science Education, $66\{1), 77-83$.

LAWSON, A.E. y RENNER, J.W., 1974, A quantitative analysis of responses to Piagetian tasks and its implications for curriculum, Science Education, 58(4), 545-559.

LAWSON, A.E., BLAKE, A. y NORDLAND, F, 1974, Piagetian tasks clarified: the use of metal cylinders, $A$ merican Biology Teacher, 36(4), 209-211.

LAWSON, A.E. y SNITGEN, D.A., 1982, Teaching formal reasoning in a college biology course for preservice teachers, Journal of Research in Science Teaching, 19(3), 233-248.

LEVINE, D.I. y LIM, M.C., 1977, Scientific reasoning ability in adolescence: Theoretical viewpoints and educational implications, Journal of Research in Science Teaching, 14(4), 371*384.

NIAZ, M., 1985a, Razonamiento Proporcional: Una interpretación basada en la teoria de Dependencia / Independencia de Campo de Witkin, Trabajo presentado en ta XXXV Convencion Anual de la Asociacion Venezolana para el Avance de la Ciencia, Mérida, Noviembre.

NIAZ, M., 1985b, Evaluation of formal operational reasoning by Venezuelan freshmen students, Research in Science and Technological Education, 3(1), 43-50.

NIAZ, M. y LAWSON, A.E., 1985, Balancing chemical equations: The role of developmental level and mental capacity, Journal of Research in Science Teaching, 22(1), $41-52$.

PASCUAL-LEONE, J., 1970, A mathematical model for the transition rule in Piaget's developmental stages, Acta Psychologica, 32, 301-345.

PIAGET, J., INHELDER, B., SZEMINSKA, A., 1960, The child's conception of geometry, (Norton: New York).

SHAYER, M. y ADEY, P., 1981, Towards a Science of Science Teaching, (Heineman: London).

WITKIN, H.A., et. al., 1962, Psychological Differentiation, (Wiley: New York).

WITKIN, H.A., GOODENOUGH, D.R. y KARP, S.A., 1967, Stability of cognitive style from childhood to young adulthood, Journal of Personality and Social Psychology, $7(3), 291-300$.

WITKIN, H.A., et al., 1971, A manual for the embedded figures tests, (Consulting Psychologists Press: Palo Alto).

WOLLMAN, W., 1977, Controlling variables: A NeoPiagetian Developmental sequence, Science Education, $61(3), 385-391$.

WOLLMAN, W. y LAWSON, A.E., 1978, The inftuence of instuction on proportional reasoning in seventh graders, Journal of Research in Science Teaching, 15(3), 227-232. 